\title{
Flexible Solar Cells Using Doped Crystalline Si Film Prepared by Self-biased Sputtering Solid Doping Source in $\mathrm{SiCl}_{4} / \mathrm{H}_{2}$ Microwave Plasma
}

Ping-Yen Hsieh, Chi-Young Lee and Nyan-Hwa Tai ${ }^{*}$

Department of Materials Science and Engineering, National Tsing Hua University,

No. 101, Section 2, Kuang-Fu Road, Hsinchu 30013, Taiwan, R.O.C.

* Corresponding author: Nyan-Hwa Tai

Postal address: Department of Materials Science and Engineering, National Tsing Hua University, No. 101, Section 2, Kuang-Fu Road, Hsinchu 30013, Taiwan, R.O.C.

Tel.: +866 $35715131 \# 42568$

Fax: + 88635737406

E-mail address: nhtai@mx.nthu.edu.tw 


\section{Supporting information}
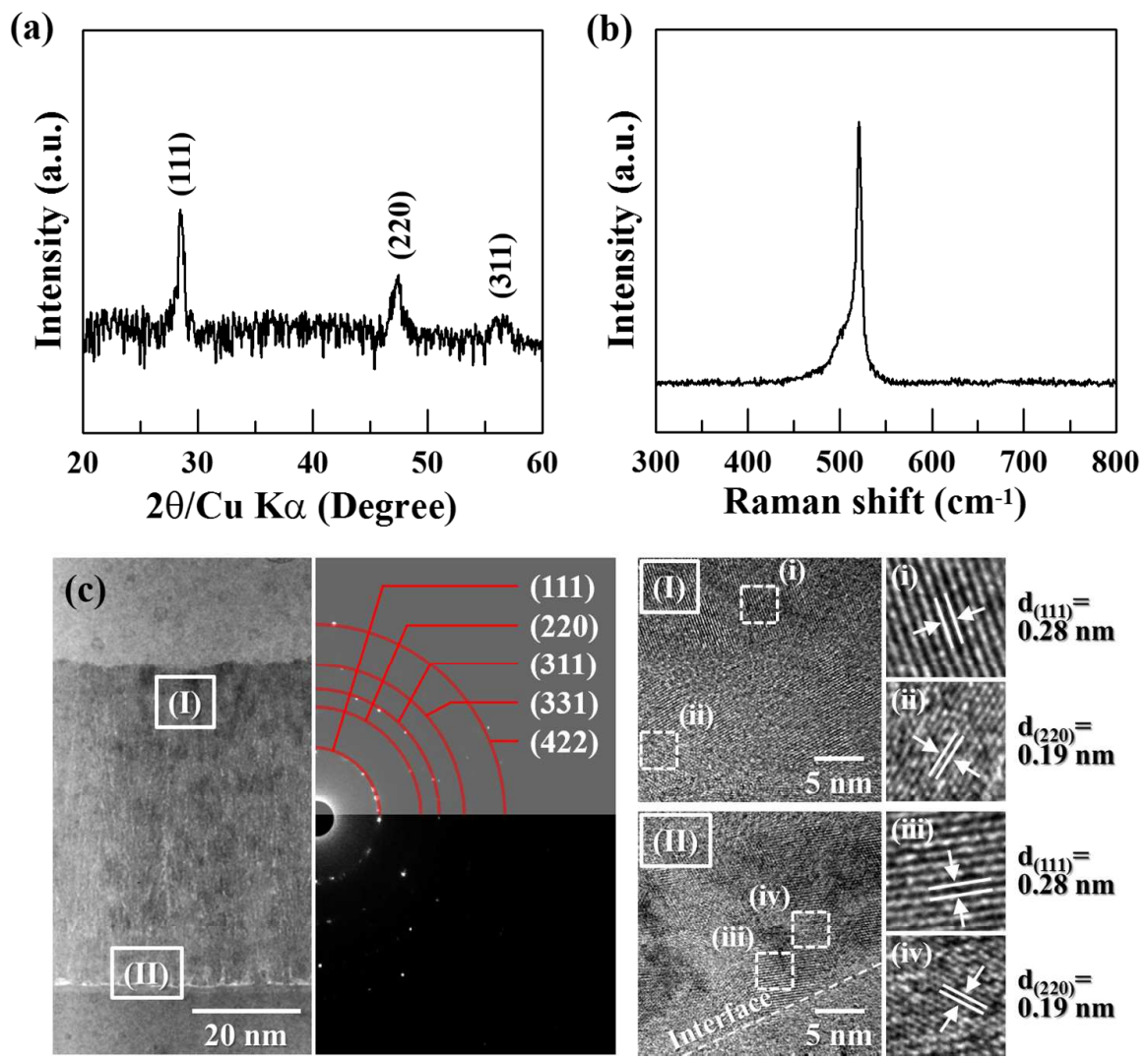

Fig. S1 (a) XRD pattern, (b) Raman spectrum, and (c) cross-sectional BFI image with SAED pattern of the P-doped Si film under condition P(6) with a thickness of $50 \mathrm{~nm}$. The HRTEM images labeled with squares (I) and (II), corresponding to the top and the bottom layers, in the BFI image are shown at the right side of (c). The enlarged areas (i) to (iv) are also inserted. 

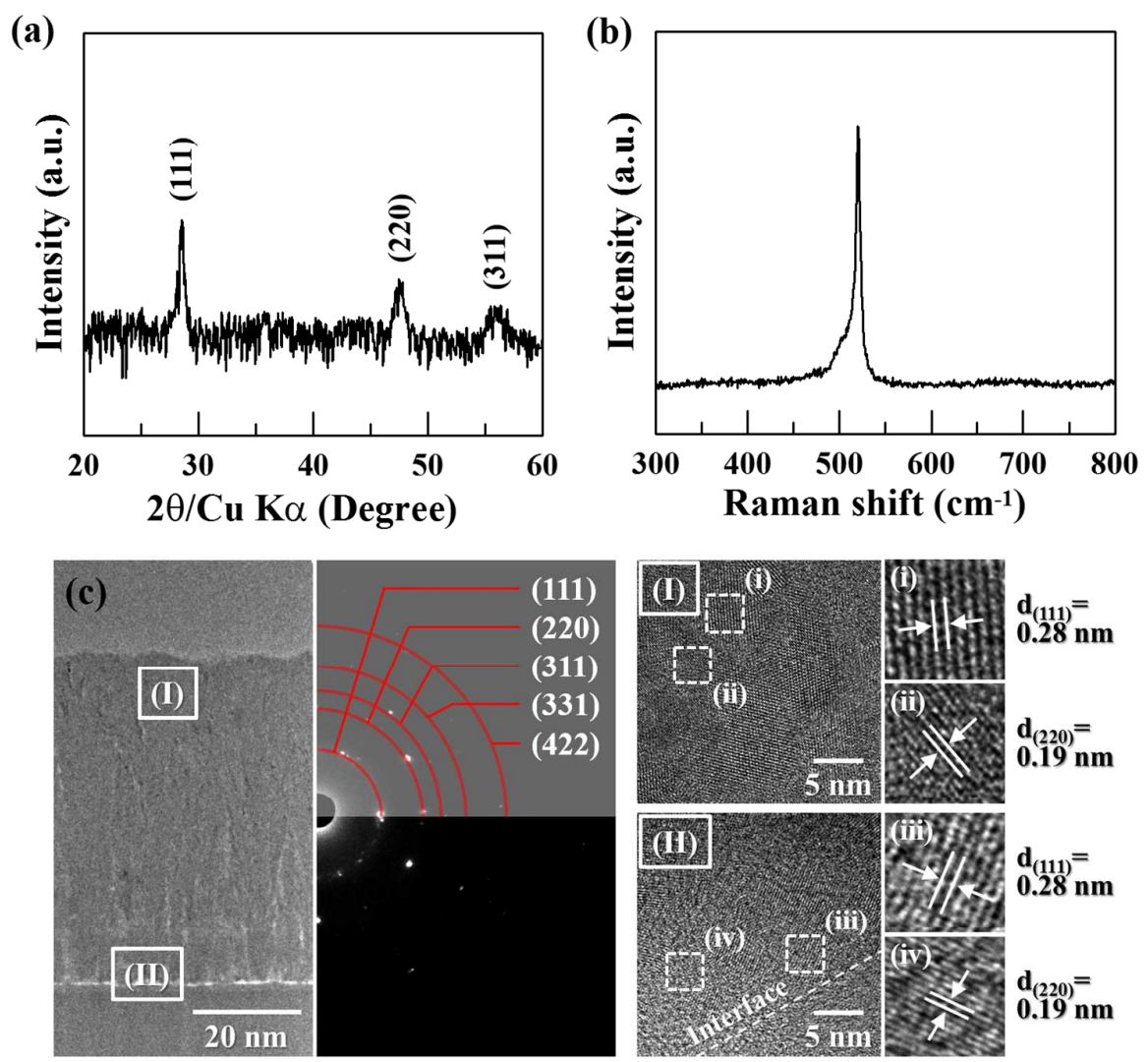

Fig. S2 (a) XRD pattern, (b) Raman spectrum, and (c) cross-sectional BFI image with SAED pattern of the B-doped Si film under condition B(10) with a thickness of $50 \mathrm{~nm}$. The HRTEM images labeled with squares (I) and (II), corresponding to the top and the bottom layers, in the BFI image are shown at the right side of (c). The enlarged areas (i) to (iv) are also inserted. 

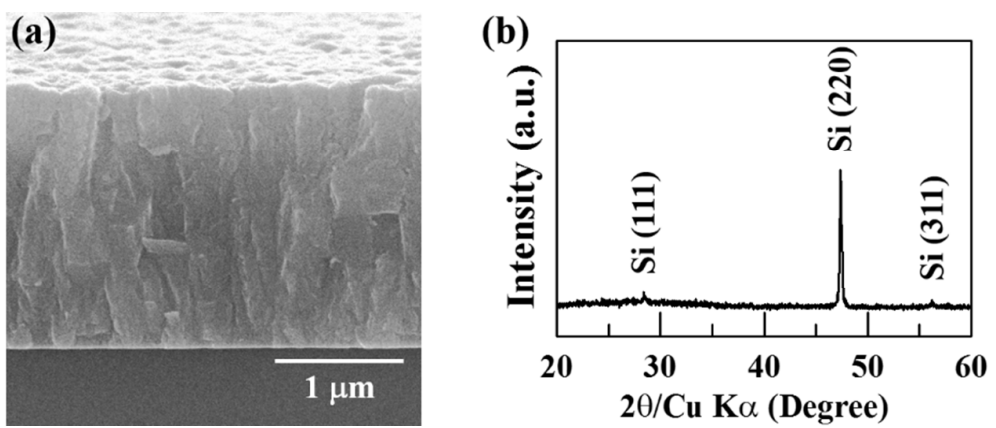

Fig. S3 (a) FESEM cross-sectional morphology and (b) XRD pattern of the intrinsic crystalline Si film with a thickness of $2 \mu \mathrm{m}$. 

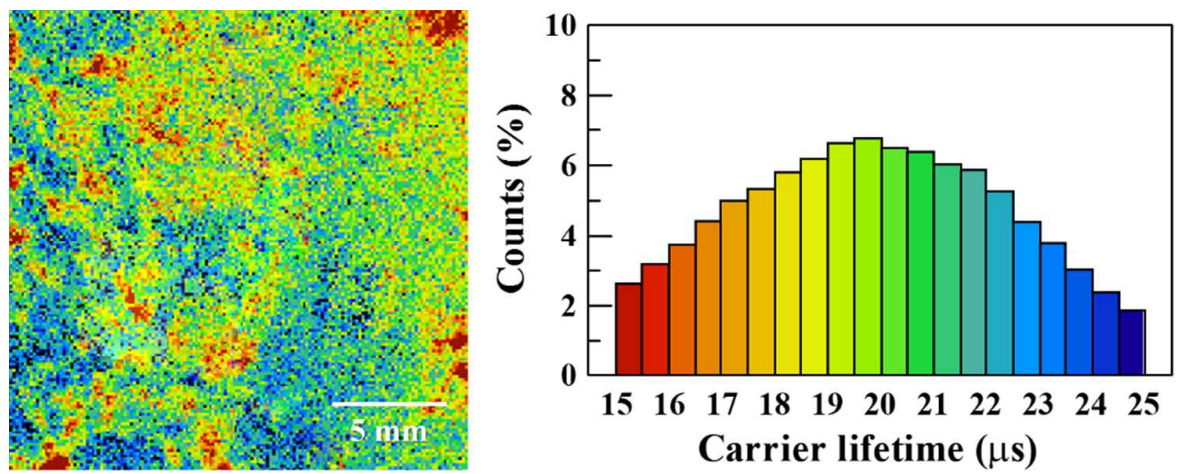

Fig. S4 Carrier lifetime mapping of the intrinsic crystalline Si film and its statistical result. 


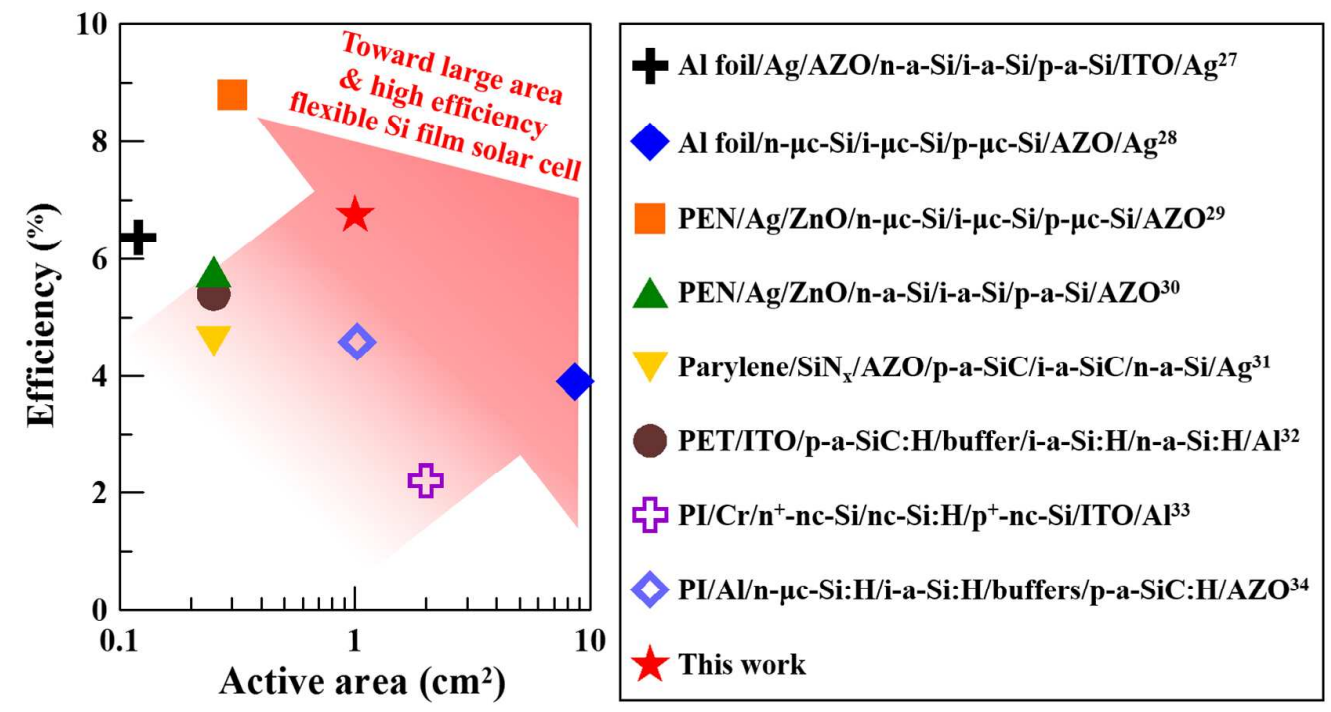

Fig. S5 Comparison of performance of flexible Si-SCs using a single junction $n-i-p$ structure. 